Pacific Journal of Mathematics

UNCONDITIONAL AND SHRINKING BASES IN LOCALLY 


\title{
UNCONDITIONAL AND SHRINKING BASES IN LOCALLY CONVEX SPACES
}

\author{
L. J. WEILL
}

Let $E$ be a locally convex space with an unconditional Schauder basis $\left\{x_{k}\right\}$ and let $\left\{f_{k}\right\}$ be the sequence of coefficient functionals biorthogonal to $\left\{x_{k}\right\}$. Owing to works of R. C. James and $\mathrm{S}$. Karlin it is known that if $E$ is a Banach space then each of the three conditions which follow is necessary and sufficient for $\left\{f_{k}\right\}$ to be a basis for $E^{*}$ in the strong (norm) topology.

(1) $E$ has no subspace topologically isomorphic to the space $l^{1}$.

(2) $E^{*}$ is separable in the strong topology.

(3) $E^{*}$ is weakly $\left(w\left(E^{*}, E^{* *}\right)\right)$ sequentially complete.

The primary purpose of this paper is to show that in certain spaces which are more general than Frechet spaces and hence than Banach spaces, each of the above three conditions is necessary and sufficient for

(0) $\left\{f_{k}\right\}$ is a strong basis for $E^{*}$.

Specifically if $E$ is a complete barrelled space, each of (1) and (2) is sufficient for (0). In any locally convex space (2) implies (1) (even if $E$ has no basis) and so each of (1) and (2) is necessary for (0). If $E$ is a space having the property that weak* bounded subsets of $E^{*}$ are strongly bounded (complete locally convex spaces and barrelled spaces have this property) then (3) is sufficient for (0). is necessary for (0) if $E$ is both barrelled and metrizable.

Besides the papers of James [8] and Karlin [9], related matter of importance is contained in the works of M. M. Day [2, Ch. 4] and C. Bessaga and A. Pelczynski [1]. E. L. Dubinsky and J. R. Retherford [5] using Köthe sequence space techniques have proved the $(1) \leftrightarrow(2)$ part of Theorem 2.12.

1. Preliminarie and fundamental theorems. Since the main results of this paper depend upon many theorems which are not widely known in their more general settings, the author thought it wise to include this section which introduces, in addition to basic theorems, some difinitions, terminology, and notation. No proofs are given for known results.

If $E$ is a locally convex space, then $\pi$ will be used for the canonical mapping from $E$ to $E^{* *}$, the space of strongly continuous functions on $E^{*}$. For convenience, a subscript 2 will be added to the word "space" to designate that the Hausdorff axiom is satisfied. 
A sequence $\left\{x_{k}\right\}$ in a linear topological space $_{2}$ is called a basis if for each element $x$ in the space there is a unique sequence of scalars $\left\{f_{k}(x)\right\}$ such that $x=\sum_{k=1}^{\infty} f_{k}(x) x_{k}$. The resulting linear functionals $\left\{f_{k}\right\}$ are called the coefficient functionals. If the coefficient functionals are continuous, then $\left\{x_{k}\right\}$ (or $\left\{x_{k}, f_{k}\right\}$ ) is called a Schauder basis. The $n$-th partial sum operator $s_{n}$ is defined by $s_{n}(x)=\sum_{k=1}^{n} f_{k}(x) x_{k}$; the $n$-th remainder is defined by $R_{n}(x)=\sum_{k=n+1}^{\infty} f_{k}(x) x_{k}$. We shall use $\Phi$ for the collection of all nonempty finite subsets of the positive integers $\omega$. If $\sigma \in \Phi$, then the $\sigma$-partial sum operator $S_{\sigma}$ is defined by $S_{\sigma}(x)=$ $\sum_{k \in \sigma} f_{k}(x) x_{k}$; the $\sigma$-remainder is defined by $R_{\sigma}(x)=x-S_{\sigma}(x)$. Note that $R_{\rho} \circ R_{\sigma}(x)=R_{e \cap \sigma}(x)$, and if $\rho \cap \sigma \neq \varnothing$ then $S_{\rho} \circ S_{\sigma}(x)=S_{\rho \cap \sigma}(x)$; if $\rho \cap \sigma=\varnothing$, then $S_{\rho} \circ S_{\sigma}(x)=\theta$ for every $x$.

Proposition 1.1. Let $E$ be a linear topological space . If $_{\text {. If }}$ $\left\{x_{k}, f_{k}\right\}$ is a Schauder basis or a weak Schauder basis for $E$, then $\left\{f_{k}, \pi\left(x_{k}\right)\right\}$ is a weak* Schauder basis for $E^{*}$. It follows that if $\left\{f_{k}\right\}$ is also a strong basis then it is a strong Schauder basis with $\left\{\pi\left(x_{k}\right)\right\}$ as the coefficient functionals. The $\sigma$-partial sum for $\left\{f_{k}\right\}$ is then the adjoint of $S_{\sigma}$; i.e., $S_{\sigma}^{*}(f)=\sum_{k \in \sigma} \pi\left(x_{k}\right)(f) f_{k}=\sum_{k \in \sigma} f\left(x_{k}\right) f_{k}$. The $\sigma$-remainder is $R_{\sigma}^{*}$ and of course $R_{\sigma}^{*} f=f-S_{\sigma}^{*} f$.

As a kind of converse we have the following.

Proposition 1.2. Let $E$ be a locally convex space. If $\left\{f_{k} F_{k}\right\}$ is a weak* Schauder basis for $E^{*}$, then $\left\{x_{k}, f_{k}\right\}$ is a weak Schauder basis for $E$, where for each $k, x_{k}$ is that unique element of $E$ such that $\pi\left(x_{k}\right)=F_{k}$.

The next two propositions require the Barrel Theorem for their proofs.

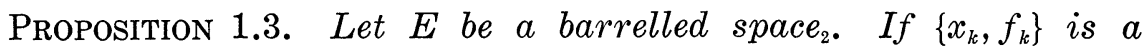
Schauder basis for $E$ then $\left\{f_{k}\right\}$ is a strong basis for the closed linear $\operatorname{span}, \overline{\left[f_{k}\right]}$, of $\left\{f_{k}\right\}$.

Actually, in Proposition 1.3, "Schauder basis" may be replaced by "weak Schauder basis" as the following reveals.

Proposition 1.4. In a barrelled space a weak Schauder basis is a Schauder basis.

A Schauder basis $\left\{x_{k}, f_{k}\right\}$ is called a shrinking basis if $\left\{f_{k}\right\}$ is a 
basis for $E^{*}$ (in the strong topology). A basis $\left\{x_{k}\right\}$ is boundedly complete if for each sequence $\left\{t_{k}\right\}$ of scalars such that $\left\{\sum_{k=1}^{n} t_{k} x_{k}\right\}_{n=1}^{\infty}$ is bounded, the series $\sum_{k=1}^{\infty} t_{k} x_{k}$ is convergent. The next proposition due to J. Dieudonné [3, Prop. 6] strengthens Proposition 1.1 in the case where $E$ is barrelled.

Proposition 1.5. If $\left\{x_{k}, f_{k}\right\}$ is a Schauder basis in a barrelled space $_{2} E$, then $\left\{f_{k}\right\}$ is a weak* boundedly complete basis for $E^{*}$. Hence, if $\left\{x_{k}, f_{k}\right\}$ is a shrinking basis then $\left\{f_{k}\right\}$ is a boundedly complete basis for $E^{*}$.

The next proposition was proved for Banach spaces by R. C. James [8] and generalized by J. R. Retherford [13].

Proposition 1.6. Let $\left\{x_{k}, f_{k}\right\}$ be a Schauder basis for a barrelled space $_{2} E$. Then $E$ is reflexive if and only if $\left\{x_{k}\right\}$ is both shrinking and boundedly complete.

If $(E,\|\cdot\|)$ is a Banach space with basis $\left\{x_{k}\right\}$, then a new norm related to the basis can be formed: $\|x\|^{\prime}=\sup _{n}\left\{\left\|S_{n}(x)\right\|\right\}$. Both norms have the same topology but the new norm is more useful. A more general device has been developed by C. W. McArthur [14]. In the fundamental lemma which follows a part of this device is abstracted even further in order that the result may be applied to Theorem 1.18.

Lemma 1.7. Let $(E, \tau)$ be a locally convex space and $\left\{g_{\alpha}: \alpha \in A\right\}$ a net $[10$, p. 28] of pointwise bounded linear operators on $E$. Let $\mathscr{V}^{-}$be the local base consisting of all barrelled neighborhoods of $E$. For $V \in \mathscr{Y}$, define $V^{\prime}$ to be $\left\{x \in E: g_{\alpha}(x) \in V\right.$ for all $\left.\alpha \in A\right\}$. Then we have the following.

(1) $\mathscr{V}^{\prime \prime} \equiv\left\{V^{\prime}: V \in \mathscr{V}\right\}$ is a local base for a locally convex topology $\tau^{\prime}$ on $E$.

(2) If there is a subnet $\left\{g_{n(\beta)}: \beta \in B\right\}$ such that, for each $x \in E,\left\{g_{n(\beta)}(x): \beta \in B\right\}$ is convergent to $x$ then $\tau^{\prime}$ is stronger than $\tau$.

(3) If in addition to (2) each $g_{\alpha}$ is continuous and $E$ is barrelled, then $\tau^{\prime}=\tau$.

Proof. By the criterion 6.5 of $\left[10\right.$, p. 47], $\mathscr{V}^{\prime}$ forms a local base for a locally convex topology if the three conditions below are met.

(i) Each $V^{\prime} \in \mathscr{Y}^{\prime \prime}$ is convex, circled, and radial at zero.

(ii) For each $U^{\prime}$ and $V^{\prime}$ of $\mathscr{V}^{\prime}$ there is a $W^{\prime} \in \mathscr{V}^{\prime \prime}$ such that 
$W^{\prime} \subset U^{\prime} \cap V^{\prime}$.

(iii) $t V^{\prime}$ belongs to $\mathscr{V}^{\prime \prime}$ for each $V^{\prime} \in \mathscr{V}^{\prime \prime}$ and each scalar $t$.

Proof of (i). Let $s$ and $t$ be nonnegative scalars such that $s+t=1$. If $x, y \in V$; then $g_{\alpha}(x), g_{\alpha}(y) \in V$ for all $\alpha \in A$. Since $V$ is convex, $s g_{\alpha}(x)+t g_{\alpha}(y)=g_{\alpha}(s x+t y) \in V$ for all $\alpha \in A$ and hence $s x+t y \in V^{\prime}$ so $V^{\prime}$ is convex. Let $|t| \leqq 1 \quad x \in V^{\prime} . g_{\alpha}(x) \in V$ for all $\alpha$ and since $V$ is circled, $t g_{\alpha}(x)=g_{\alpha}(t x) \in V$ for all $\alpha$; hence $t x \in V^{\prime}$. Therefore $V^{\prime}$ is circled. Let $x \in E$. Since $g_{\alpha}$ is pointwise bounded, there is a positive scalar $s$ such that if $0<t<s$ then $\operatorname{tg}_{\alpha}(x) \in V$ for all $\alpha$. Hence $t x \in V^{\prime}$ so $V^{\prime}$ is radial at zero.

Proof of (ii). There exists a $W \in \mathscr{Y}^{-}$such that $W \cup U \cap V$. Hence $W^{\prime} \subset(U \cap V)^{\prime}=U^{\prime} \cap V^{\prime}$.

Proof of (iii). For each scalar $t, t V \in \mathscr{Y}^{\prime}$. Hence $(t V)^{\prime}=t V^{\prime} \in \mathscr{Y}^{\prime}$.

To prove (2) we show that $V^{\prime} \subset V$ for each $V \in \mathscr{Y}^{\prime}$. If $x \in V^{\prime}$, then $g_{\alpha}(x) \in V$ for all $\alpha$ and in particular $g_{n(\beta)}(x) \in V$ for all $\beta \in B$. Thus since $V$ is closed $V$ is closed $x \in V$.

To prove (3) we show that $V^{\prime}$ is $\tau$-closed and hence a $\tau$-barrel. Let $\left\{y_{\beta}: \beta \in B\right\}$ be a net in $V^{\prime}$ converging to $y$. Let $\alpha \in A$; then since $g_{\alpha}$ is continuous, $g_{\alpha}\left(y_{\beta}\right)$ converges with $\beta$ to $g_{\alpha}(y)$. Since $g_{\alpha}\left(y_{\beta}\right) \in V$ for each $\beta \in B, g_{\alpha}(y) \in V$; $\alpha$ was arbitrary, so $y \in V^{\prime}$.

Part (1) and (2) of the next proposition follow from the lemma.

Proposition 1.8. Let $(E, \tau)$ be a locally convex space with $^{2}$ basis $\left\{x_{k}\right\}$ and let $\mathscr{Y}^{-}$be as in Lemma 1.7. For $V \in \mathscr{Y}^{-}$, define $V^{\prime}=\left\{x: S_{n}(x) \in V\right.$ for each $\left.n \in \omega\right\}$.

(1) $\mathscr{Y}^{\prime \prime}=\left\{V^{\prime}: V \in V\right\}$ is a local base for a locally convex topology $\tau^{\prime}$ which is stronger than $\tau$.

(2) If $E$ is barrelled and $\left\{x_{k}\right\}$ is a Schauder basis then $\tau=\tau^{\prime}$.

(3) If $(E, \tau)$ is complete, then so is $\left(E, \tau^{\prime}\right)$.

The last propositions of this section are slight modifications of results due to Retherford and McArthur [14],

Proposition 1.9. Let $E$ be a complete locally convex space s $_{2}$ and $\left\{x_{k}\right\}$ a sequence of nonzero elements of $E$. If for each continuous seminorm $P$ on $E$ there is a continuous seminorm $Q$ such that $P\left(\sum_{k=1}^{p} a_{k} x_{k}\right) \leqq Q\left(\sum_{k=1}^{q} a_{k} x_{k}\right)$ for $p \leqq q$ and arbitrary scalars $a_{1}, \cdots, a_{q}$, then $\left\{x_{k}\right\}$ is a Schauder basic sequence (i.e., $\left\{x_{k}\right\}$ is a Schauder basis for its closed linear span $\left[\overline{\left[x_{k}\right]}\right]$.

Proposition 1.10. Let $E$ be a barrelled space ${ }_{2}$ with a Schauder basis $\left\{x_{k}\right\}$. Then for each continuous seminorm $P$ there is a continuous seminorm $Q$ such that for $p \leqq q$ and arbitrary scalars 
$a_{1}, \cdots, a_{q}$, we have $P\left(\sum_{k=1}^{p} a_{k} x_{k}\right) \leqq Q\left(\sum_{k=1}^{q} a_{k} x_{k}\right)$.

Let $\sum_{k=1}^{\infty} y_{k}$ be a (formal) series in a linear topological space $E$. The series is called unordered bounded if the set of unordered partial sums $\left\{\sum_{k \infty o} y_{k}: \sigma \in \phi\right\}$ is bounded. The series is called unconditionally (or unordered) convergent if the net of unordered partial sums (with inclusion as the order relation in $\phi$ ) is convergent to an element of $E$. It is well known that a series $\sum_{k=1}^{\infty} y_{k}$ in a linear topological space $_{2}$ is unconditionally convergent if and only if for each permutation $p$ of $\omega$, the set of positive integers, the series $\sum_{k=1}^{\infty} y_{p}(k)$ is convergent (each series converges to the same element regardless of the permutation).

The series $\sum_{k=1}^{\infty} y_{k}$ is subseries convergent if for every subsequence $\left\{y_{n_{k}}\right\}$ of $\left\{y_{k}\right\}$ the series $\sum_{k=1}^{\infty} y_{n_{k}}$ is convergent. $\sum_{k=1}^{\infty} y_{k}$ is boundedmultiplier convergent if for each bounded sequences of scalars $\left\{t_{k}\right\}$ the series $\sum_{k=1}^{\infty} \mathrm{t}_{k} y_{k}$ is convergent. $\sum_{k=1}^{\infty} y_{k}$ is absolutely convergent in a locally convex space $E$ if the series is convergent and for each continuous seminorm $P$ on $E$, the series $\sum_{k=1}^{\infty} P\left(y_{k}\right)$ is convergent. In any locally convex space, absolute convergence implies unconditional convergence.

Proposition 1.11. In a locally convex space a series is weakly absolutely convergent if and only if it is weakly unconditionally convergent.

Define $a$ to be the set of sequences $\left\{\alpha_{m}\right\}$ such that $\alpha_{m}= \pm 1$ for each $m$; define $b$ to be the set of sequences $\left\{\beta_{m}\right\}$ such that $\beta_{m}=0$ or 1 for each $m$; and define $e$ to be the set of all complex sequences $\left\{\varepsilon_{m}\right\}$ such that $\left|\varepsilon_{m}\right| \leqq 1$ for each $m$.

Proposition 1.12. If $\sum_{k=1}^{\infty} y_{k}$ is a series in a locally convex space $E$, then the statements below are equivalent.

(1) $\sum y_{k}$ is unordered bounded.

(2) $\sum y_{k}$ is weakly unordered Cauchy.

(3) The set $\left\{\sum_{k=1}^{n} \alpha_{k} y_{k}:\left\{\alpha_{k}\right\} \in a\right.$ and $\left.n \in \omega\right\}$ is bounded.

(4) The set $\left\{\sum_{k=1}^{n} \beta_{k} y_{k}:\left\{\beta_{k}\right\} \in b\right.$ and $\left.n \in \omega\right\}$ is bounded.

(5) The set $\left\{\sum_{k=1}^{n} \varepsilon_{k} y_{k}:\left\{\varepsilon_{k}\right\} \in e\right.$ and $\left.n \in \omega\right\}$ is bounded.

COROLLARY 1.13. In a weakly sequentially complete locally convex space a series is unordered bounded if and only if it is weakly unconditionally convergent (weakly absolutely convergent).

The Orlicz-Pettis theorem for Banach spaces on subseries convergence has been shown valid for locally convex spaces by Grothendieck 
and McArthur. For a proof of the theorem, which follows, see [12].

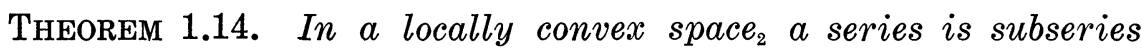
convergent if and only if it is weakly subseries convergent.

It is well known that in a sequentially complete locally convex space, bounded-multiplier convergence and subseries convergence are each equivalent to unconditional convergence [2]. The next theorem adds three other conditions which are equivalent to unconditional convergence. A proof of the equivalence of (1), (2), (3), and (4) can be found in [15]. (1) is proved equivalent to (2) in [11] by use of a certain convergence criterion.

THEOREM 1.15. Let $\left\{y_{k}\right\}$ be a sequence in a sequentially complete locally convex space $E$. The following six statements are equivalent.

(1) The series $\sum_{k=1}^{\infty} y_{k}$ converges unconditionally.

(2) For any equicontinuous subset $A$ of $E^{*}$, the series $\sum_{k=1}^{\infty}\left|f\left(y_{k}\right)\right|$ converges uniformly for $f \in A$.

(3) The series $\sum_{k=1}^{\infty} \alpha_{k} y_{k}$ converges uniformly for $\left\{\alpha_{k}\right\} \in a$.

(4) The series $\sum_{k=1}^{\infty} \varepsilon_{k} y_{k}$ converges uniformly for $\left\{\varepsilon_{k}\right\} \in e$.

(5) The series $\sum_{k=1}^{\infty} y_{k}$ is bounded-multiplier convergent.

(6) The series $\sum_{k=1}^{\infty} y_{k}$ is subseries convergent.

Since a weakly (sequentially) complete locally convex space is (sequentially) complete, Theorem 1.14 and 1.15 imply that in a weakly sequentially complete locally convex space a series is unconditionally convergent if and only if it is weakly unconditionally convergent. Thus similarly to Corollary 1.13 we have the following.

COROLlary 1.16. In a weakly sequentially complete locally convex space $_{2}$, a series is unordered bounded if and only if it is unconditionally convergent.

A basis $\left\{x_{k}, f_{k}\right\}$ in a linear topological space $_{2}$ is called an unconditional basis if for each $x$ in the space the series $\sum_{k=1}^{\infty} f_{k}(x) x_{k}$ is unconditionally convergent to $x$. Hence, $\left\{x_{k}, f_{k}\right\}$ is an unconditional Schauder basis if and only if for each permutation $p$ of $\omega,\left\{x_{p(k)}, f_{p(k)}\right\}$ is a Schauder basis. It follows that each of Propositions 1.1 and 1.5 has an analog for unconditional bases. For instance we have the following.

THEOREM 1.17. If $\left\{x_{k}, f_{k}\right\}$ is an unconditional Schauder basis for a linear topological space $2 E$, then $\left\{f_{k}, \pi\left(x_{k}\right)\right\}$ is a weak* unconditional Schauder basis for $E^{*}$. 
If $\left\{x_{k}, f_{k}\right\}$ is a basis and $\alpha=\left\{\alpha_{k}\right\}$ is a sequence of scalars, define $S_{n, \alpha}(x)=\sum_{k=1}^{n} \alpha_{k} f_{k}(x) x_{k}$. Let $(E, \tau)$ be a locally convex space ${ }_{2}$ with an unconditional Schauder basis $\left\{x_{k}, f_{k}\right\}$. Let $\mathscr{Y}^{-}$be the local base consisting of all barrelled neighborhoods in $E$. If $V \in \mathscr{V}^{-}$, define $V_{b}=\left\{x \in E: S_{o}(x) \in V\right.$ for all $\left.\sigma \in \Phi\right\}, \quad V_{a}=\left\{x \in E: S_{n, \alpha}(x) \in V\right.$ for all $n \in \omega$ and all $\alpha \in a\}$, and $V_{e}=\left\{x \in E: S_{n, \varepsilon}(x) \in V\right.$ for all $n \in \omega$ and all $\varepsilon \in e\}$. Let $\mathscr{V}_{b}^{-}=\left\{V_{b}: V \in \mathscr{Y}^{-}\right\}, \quad \mathscr{V}_{a}^{-}=\left\{V_{a}: V \in \mathscr{V}^{-}\right\}$, and $\mathscr{V}_{e}^{-}=$ $\left\{V_{e}: V \in \mathscr{Y}\right\}$.

Theorem 1.18. Le $(E, \tau)$ be as above. The families $\mathscr{Y}_{b}^{-}, \mathscr{Y}_{a}$, and $\mathscr{V}^{-}$form local bases for locally convex Hausdorff topologies on $E$, say $\tau_{b}, \tau_{a}$, and $\tau_{e}$. Each of these topologies is stronger than $\tau$ and if $E$ is barrelled then $\tau=\tau_{b}=\tau_{a}=\tau_{e}$.

Proof. According to Lemma 1.7, $\mathscr{Y}_{b}, \mathscr{Y}_{a}$, and $\mathscr{Y}_{e}$ form local bases if the families of operators $\left\{S_{\sigma}: \sigma \in \Phi\right\},\left\{S_{n, \alpha}:(n, \alpha) \in \omega \times a\right\}$, and $\left\{S_{n, \varepsilon}:(n, \varepsilon) \in \omega \times e\right\}$ are pointwise bounded. Since $\left\{x_{k}, f_{k}\right\}$ is an unconditional Schauder basis it is a weak unconditional Schauder basis. Thus $\sum_{k=1}^{\infty} f_{k}(x) x_{k}$ is weakly unordered Cauchy for each $x$ and hence by Proposition 1.12 the three families above are pointwise bounded.

Let $d$ be either the family of sequences $a$ or $e$. The set $\omega \times d$ is a directed set under the order relation $<$ defined by: $(n, \alpha)<(m, \beta)$ if and only if $n \leqq m$. Thus $\left\{S_{n, \alpha}:(n, \alpha) \in \omega \times d\right\}$ is a net. Let $\alpha^{1}$ be the sequence $(1,1,1, \cdots)$ and not that the subnet $\left\{S_{n, \alpha^{1}}(x): n \in \omega\right\}$ converges to $x$ for each $x \in E$. Thus by part (2) of 1.7. $\tau_{b}, \tau_{a}$, and $\tau_{e}$ are stronger than $\tau$.

Let $\mathscr{Y}^{\prime \prime}$ be $\mathscr{Y}_{b}, \mathscr{V}_{a}{ }_{a}$, or $\mathscr{V}_{e}^{-}$then $\bigcap_{V^{\prime} \in \mathscr{Y}}, V^{\prime} \subset \bigcap_{V \in \mathscr{Y}} V$. Since $\tau$ is a Hausdorff topology, the latter intersection is empty and hence the other topologies are also Hausdorff.

For each $n \in \omega$ and $\alpha$ in $b, a$, or $e$, the operator $S_{n, \alpha}$ is continuous so if $E$ is barrelled then $\tau=\tau_{b}=\tau_{a}=\tau_{e}$.

The next result, its variations, and its corollary shall be referred to as the Generalized Gurevic Theorem (see [7]). We use $d$ to mean either of the family of sequences $b, a$, or $e$ (actually the theorem is valid if $d$ satisfies $a \subset d \subset e$ or $b \subset d \subset e)$.

THEOREM 1.19. Let $\left\{x_{k}\right\}$ be a sequence of nonzero elements in a locally convex space ${ }_{2} E$. Call the following condition*: given a neighborhood of zero $U$ there is a neighborhood of zero $V$ such that for an arbitrary sequence $\left\{a_{n}\right\}$ of scalars arbitrary $p$ and $q$ in $\{k: k$ is a positive integer or $k=\infty\}$ with $q \geqq p$, and arbitrary sequence $\left\{\delta_{n}\right\} \in d$, we have $\sum_{n=1}^{q} a_{n} x_{n} \in V$ implies $\sum_{n=1}^{p} \delta_{n} a_{n} x_{n} \in U$. 
(1) If $E$ is sequentially complete, then $*$ implies $\left\{x_{k}\right\}$ is unconditionally Schauder basic.

(2) If $E$ is barrelled and $\left\{x_{k}\right\}$ is an unconditional Schauder basis for $E$, then * holds.

VARIATION 1.20. In the theorem, condition $*$ can be replaced by condition $*_{1}$ : given an equicontinuous set $A \subset E^{*}$, there is an equicontinuous set $B \subset E^{*}$ such that for an arbitrary positive number $M$ and arbitrary sequence $\left\{a_{n}\right\}$ of scalars arbitrary $p$ and $q$ in $\{k: k$ is a positive interger or $k=\infty\}$ with $q \geqq p$, and arbitrary sequence $\left\{\delta_{n}\right\} \in d$, we have $\left|\sum_{n=1}^{q} a_{n} g\left(x_{n}\right)\right| \leqq M$ for all $g \in B$ implies $\left|\sum_{n=1}^{p} \delta_{n} a_{n} f\left(x_{n}\right)\right| \leqq M$ for all $f \in A$.

VARIATION 1.21. In the theorem condition * can be replaced by condition $*_{2}$ : given a continuous seminorm $P$ there is a continuous seminorm $Q$ such that for arbitrary sequence $\left\{a_{n}\right\}$ of scarlars arbitrary $p$ and $q$ in $\{k: k$ is a positive integer or $K=\infty\}$ with $q \geqq p$, and arbitrary sequence $\left\{\delta_{n}\right\} \in d$ we have $P\left(\sum_{n=1}^{p} \delta_{n} a_{n} x_{n}\right) \leqq Q\left(\sum_{n=1}^{q} a_{n} x_{n}\right)$.

Proof. First we show that $*$ and $*_{1}$ are equivalent. Since $\left\{A_{0}: A\right.$ equicontinuous $\}$ forms a local base in $E, *$ is easily seen to be equivalent to the following. Given an equicontinuous $A$ there is an equicontinuous $B$ such that for $\ldots$ we have $\sum_{n=1}^{q} a_{n} x_{n} \in B_{0}$ implies $\sum_{n=1}^{p} \delta_{n} a_{n} x_{n} \in A_{0}$. But this is equivalent to $*_{1}$.

Now we show that $*$ and $*_{2}$ are equivalent. Suppose that $*_{2}$ holds. Let $U$ be a barrelled neighborhood of zero and let $P$ be the Minkowski functional of $U$ ( $U$ equals $U_{P}$, the closed unit ball of $P$ ). In accordance with $*_{2}$ corresponding to $P$ there is a continuous seminorm $Q$ such that $P\left(\sum^{p}\right) \leqq Q\left(\sum^{q}\right)$. If $\Sigma^{q} \in U_{Q}$ then $Q\left(\sum^{q}\right) \leqq 1$ and hence $P\left(\Sigma^{p}\right) \leqq 1$; that is, $\sum^{p} \in U_{P}$. To show that $*$ implies $*_{2}$ let $P$ be a continuous seminorm and $U_{P}$ its unit ball. There exists a $U_{Q}$ (i.e., a barrelled neighborhood of zero with Minkowski functional $Q$ ) such that $\Sigma^{q} \in U_{Q}$ implies $\Sigma^{p} \in U_{P}$; or $Q \Sigma^{q} \leqq \varepsilon$ implies $P \sum^{p} \leqq \varepsilon$. If $Q \sum^{q} \equiv Q \sum_{k=1}^{q} a_{n_{k}} x_{n_{k}}$ is zero then $P \sum^{p} \leqq \varepsilon$ for every $\varepsilon>0$ so that $P \sum^{p}=0$. Hence $Q \sum^{q}=P \sum^{p}=0$. If $Q \sum^{q} \neq 0$ then let $t=1 / Q \sum^{q} . \quad Q\left(t \sum^{q}\right)=1$; hence $P\left(t \sum^{p}\right) \leqq 1$. Upon substitution for $t$ we get $P\left(\sum^{p}\right) \leqq Q\left(\sum^{q}\right)$.

To prove part (1) of the theorem, observe that $*_{2}$ is a stronger condition than that in Proposition 1.9 and hence $\left\{x_{k}\right\}$ is a Schauder basic sequence. To show that $\left\{x_{k}\right\}$ is unconditional, let $U$ be a neighborhood of zero in $E$. In accordance with $*$ let $V$ be the neighborhood corresponding to $U$. Let $\left\{f_{k}\right\} \subset \overline{\left[x_{k}\right]} *$ be the family of coefficient functionals for $\left\{x_{k}\right\}$. Let $x \in \overline{\left[x_{k}\right]}$; then for $n$ and $m$ large enough $\sum_{k=m}^{n} f_{k}(x) x_{k} \in V$. Hence if $\left\{\delta_{k}\right\}$ is any element of $d$, 
$\sum_{k=m}^{n} \delta_{k} f_{k}(x) x_{k} \in U$. It follows from Theorem 1.15 and the completeness of $E$ that $\sum_{k=1}^{\infty} f_{k}(x) x_{k}$ is unconditionally convergent.

Part (2) is an immediate consequence of Theorem 1.18. Given a barrelled neighborhood of zero $U$, then $U_{d} \equiv\left\{x \in E: S_{n, \delta}(x) \in U\right.$ for all $n \in \omega$ and $\delta \in d\}$ is also a neighborhood of zero, and $U_{d}$ satisfies the requirements for $V$ in $*$.

COROLlaRY 1.22. Let $E$ be a barrelled space with $_{2}$ unconditional Schauder basis $\left\{x_{k}, f_{k}\right\}$ and let $\left\{y_{k}\right\}$ be any sequence in $E$ and $\left\{\sigma_{k}\right\}$ a sequence in $\Phi$ such that $\sigma_{n} \cap \sigma_{m}=\varnothing$ if $m \neq n$. Define $w_{k}$ to be the $\sigma_{k}$-partial sum of $y_{k}$; i.e., $w_{k}=\sum_{j \in \sigma_{k}} f_{j}\left(y_{k}\right) x_{j}$. Then given a neighborhood of zero $U$ there is a neighborhood of zero $V$ such that for arbitrary scalars $t_{k}$, arbitrary $\left\{\delta_{k}\right\} \in d$, and arbitrary $n$ and $m$ in $\{k: k \in \omega$ or $k=\infty\}$ with $n \geqq m$ we have $\sum_{k=1}^{n} t_{k} w_{k} \in V$ implies $\sum_{k=1}^{m} \delta_{k} t_{k} w_{k} \in U$. Thus if $E$ is also sequentially complete and for each $k, w_{k} \neq 0$ then the sequence $\left\{w_{k}\right\}$ is unconditionally basic in $E$.

Proof. Simply observe that if $a_{j k}=t_{k} f_{j}\left(y_{k}\right)$, then $\sum_{k=1}^{n} t_{k} w_{k}=$ $\sum_{j \in \sigma_{k}, k \in\left\{1,2, \ldots,{ }_{n}\right\}} a_{j k} x_{j}$ and in the expansion the $j$ 's are all distinct by the null intersection requirement of the hypothesis. Then apply Theorem 1.19.

The Generalized Gurevic Theorem, which we have just proved, is of fundamental importance. We shall apply it often in the next section.

2. Unconditional and shrinking bases. As pointed out in the introductory remarks, we shall show that most of the results of James and Karlin are valid for complete barrelled spaces, barrelled spaces, or complete locally convex spaces. Even unqualified locally convex spaces are sufficient for a few of the more simple results. The first theorem follows easily from the remarks made after the definition of unconditional basis, page 12, and Proposition 1.3.

THEOREM 2.1. Let $E$ be a barrelled space with an unconditional basis $\left\{x_{k}, f_{k}\right\}$. If $\left\{x_{k}, f_{k}\right\}$ is shrinking, then $\left\{f_{k} \pi\left(x_{k}\right)\right\}$ is an unconditional Schauder basis for $E^{*}$.

Hereafter we shall use " $\omega$-complete" for "sequentially complete".

Lemma 2.2. Let $E$ be a locally convex space ${ }_{2}$ and $\left\{y_{k}\right\}$ a bounded sequence in $E$.

(1) If $E$ is $\omega$-complete, then the series $\sum_{k=1}^{\infty} t_{k} y_{k}$ is convergent for each $t=\left\{t_{k}\right\} \in l^{1}$. 
(2) If $F$ is a subspace of $l^{1}$ such that for each $t \in F$ the series $\sum_{k=1}^{\infty} t_{k} y_{k}$ is convergent, then the linear mapping $T: F \rightarrow E$ defined by $T(t)=\sum_{k=1}^{\infty} t_{k} y_{k}$ is continuous.

Proof. To see that $\sum_{k=1}^{\infty} t_{k} y_{k}$ is convergent if $E$ is $\omega$-complete, let $A_{0}$ be a neighborhood of zero (where $A$ is an equicontinuous subset of $\left.E^{*}\right)$. Since $\left\{y_{k}\right\}$ is bounded there is a positive scalar $s$ such that $y_{k} \in s A_{0}$ for each $k$. For $n$ and $m$ large enough $\sum_{k=m}^{n}\left|t_{k}\right|<1 / s$. Since $\left|f\left(y_{k}\right)\right| \leqq s$ for each $f \in A$ and $k \in \omega$ it follows that $\sum_{k=m}^{n} t_{k} y_{k} \in A_{0}$. Hence the series is convergent.

Let $A$ be equicontinuous and define $M=\sup \left\{\left|f\left(y_{k}\right)\right|: f \in A, k \in \omega\right\}$. If $t \in F$ and $\|t\|<1 / M+1$ then for $f \in A,|f T(t)|=\sum_{k=1}^{\infty} t_{k} f\left(y_{k}\right) \mid \leqq$ $\sum_{k=1}^{\infty}\left|t_{k}\right| \cdot\left|f\left(y_{k}\right)\right|<1$. Therefore $T(t) \in A_{0}$ so $T$ is continuous.

Lemma 2.3. Let $T$ be a linear transformation of a normed space $E$ into a locally convex space $F$. If there exists a continuous functional $f$ on $F$ such that $f(\theta)=0$ and a positive number $m$ such that $|f T(x)| \geqq m\|x\|$ for each $x \in E$, then $T$ is one-to-one and relatively open.

Proof. If $T(x)$ is zero then so is $f(T(x))$ and hence by the given inequality, $x=\theta$; so $T$ is one-to-one. To prove that $T$ is relatively open we show that the inverse map $T^{-1}: T(E) \rightarrow E$ is bounded on a neighborhood of zero, vis. $f^{-1}[-1,1] \cap T(E)$. Let $x \in T^{-1}\left(f^{-1}[-1,1]\right)$ then $T(x) \in f^{-1}[-1,1]$ so that $|f T(x)| \leqq 1$. Using the given inequality we get $m\|x\| \leqq|f T(x)| \leqq 1$. Thus $\|x\| \leqq 1 / m$ for all $x \in T^{-1}\left(f^{-1}[-1,1]\right)$; i.e., $T^{-1}\left(f^{-1}[-1,1]\right)$ is bounded and hence $T^{-1}: T(E) \rightarrow E$ is continuous.

Lemma 2.4. Let $E$ be a locally convex space and $F$ a subspace of $E$. If $E^{*}$ is separable, then so is $F^{*}$ (in the $s\left(F^{*}, F\right)$ topology).

Proof. Let $F^{1}=\left\{f \in E^{*}: f(x)=0\right.$ for each $\left.x \in F\right\}$. The continuous image of a separable topological space is separable. Thus each quotient space (with quotient topology) of a separable space is separable. Hence $E^{*} / F^{1}$ is separable. The canonical map $[f] \rightarrow f / F$ is an isomorphism mapping $E^{*} / F^{1}$ onto $F^{*}$ (see [10, p. 120]). Furthermore this mapping is continuous (where $E^{*} / F^{1}$ has the quotient topology and $F^{*}$ the $s\left(F^{*}, F\right)$ topology) as we now show. It suffices to show that the mapping $\phi: E^{*} \rightarrow F^{*}$ defined by $\phi(f)=f / F$ is continuous since this map is the composition of the quotient map $f \rightarrow[f]$ and the mapping $[f] \rightarrow f / F$ (see [10, Th. 5.7, p. 39]). If $A \subset F$ then denote by $A^{\circ F^{*}}$ the polar of $A$ in $F^{*}$ and use $A^{\circ E^{*}}$ for the polar of $A$ in $E^{*}$. Let $B$ be a bounded subset of $F$. Then it is not hard to see that $\phi^{-1}\left[B^{\circ F^{*}}\right]=B^{\circ E^{*}}$ and hence $\phi$ is continuous. Thus the canonical 
mapping from $E^{*} / F^{1}$ onto $F^{*}$ is continuous and therefore $F^{*}$ is separable.

Define a non- $l^{1}$ space (non- $c_{0}$ space) to be a locally convex space which has no subspace topologically isomorphic to $l^{1}\left(c_{0}\right)$.

CoRollary 2.5. If $E$ is a locally convex space and $E^{*}$ is separable, then $E$ is a non-l $l^{1}$ space.

Proof. If $E$ contains a subspace $F$ topologically isomorphic to $l^{1}$, then $F^{*}$ is topologically isomorphic to $m$, the nonseparable space of bounded sequences. Thus by Lemma 2.4, $E^{*}$ could not be separable.

The following is an immediate consequence of the corollary.

THEOREM 2.6. Let $E$ be a locally convex space sith $_{2}$ basis $\left\{x_{k}, f_{k}\right\}$. If $\left\{x_{k}, f_{k}\right\}$ is shrinking, then $E$ is a non-l $l^{1}$ space.

To get a converse of Theorem 2.6, the space and basis must be strengthened.

THEOREM 2.7. Let $E$ be a $\omega$-complete barrelled space ${ }_{2}$ with an unconditional Schauder basis $\left\{x_{k}, f_{k}\right\}$. If $E$ is a non-l $l^{1}$ space, then $\left\{x_{k}, f_{k}\right\}$ is a shrinking basis.

Proof. Suppose that $\left\{x_{k}, f_{k}\right\}$ is not a shrinking basis. Then for some $g \in E^{*}$ the series $\sum_{k=1}^{\infty}\left[\pi\left(x_{k}\right)\right](g) f_{k}=\sum_{k=1}^{\infty} g\left(x_{k}\right) f_{k}$ is not strongly convergent. Thus since the series is weak* convergent (Proposition 1.1) it can not be strongly Cauchy. Therefore there is a strong neighborhood of zero $B^{\circ}$ ( $B$ is bounded in $E$ ) such that for each positive integer $j$ there are integers $n_{j}$ and $m_{j}, n_{j}>m_{j}>j$, with the property that $\sum_{k=m_{i}}^{n_{j}} g\left(x_{k}\right) f_{k} \notin B^{0}$; furthermore, the sequence $\left\{n_{j}\right\}_{j=1}^{\infty}$ and $\left\{m_{j}\right\}_{j=1}^{\infty}$ can be chosen so that $m_{j+1}>n_{j}$ for each $j$. This means that for each $j$ there is a $z_{j} \in B$ with the property that $\left|\sum_{k=m}^{n_{j}} g\left(x_{k}\right) f_{k}\left(z_{j}\right)\right|>1$; or, if we let $\left[m_{j}, n_{j}\right]$ denote just the integers between $m_{j}$ and $n_{j}$ then $\left|g S\left(z_{j}\right)_{\left[m_{j}, n_{j}\right]}\right|>1$. Since the family $\left\{S_{\left.\left[m_{j}, n_{j}\right]\right\}_{j=1}^{\infty}}\right.$ is pointwise bounded (Proposition 1.12) it is equicontinuous because $E$ is barrelled. Hence since $\left\{z_{j}\right\}$ is bounded, the set $\left\{S\left(z_{j}\right)_{\left[m_{j}, n_{j}\right\}^{\infty}}\right.$ is bounded in $E$. If we let $y_{j}=S\left(z_{j}\right)_{\left[m_{j}, n_{j}\right]}$ then we have shown that for each $j,\left|g\left(y_{j}\right)\right|>1$ and the sequence $\left\{y_{j}\right\}$ is bounded. Let $t=\left\{t_{j}\right\} \in l^{1}$ and $t \neq 0$ and chooose the scalars $\varepsilon_{j}$ so that $\left|t_{j} g\left(y_{j}\right)\right|=\varepsilon_{j} t_{j} g\left(y_{j}\right)$. By Lemma 2.2 the series $\sum_{k=1}^{\infty} t_{k} y_{k}$ is convergent and by the Generalized Gurevic Theorem (Corollary 1.22) the 
sequence $\left\{y_{j}\right\}$ is unconditionally basic. Therefore the series $\sum_{j=1}^{\infty} \varepsilon_{j} t_{j} y_{j}$ is convergent (Theorem 1.12). Now we have $\left|g \sum_{j=1}^{\infty} \varepsilon_{j} t_{j} y_{j}\right|=$ $\sum_{j=1}^{\infty}\left|t_{j}\right| \cdot\left|g\left(y_{j}\right)\right|>\sum_{j=1}^{\infty}\left|t_{j}\right|$. In accordance with Variation 1.20 let $A$ be the equicontinuous subset of $E^{*}$ which depends upon the given equicontinuous set $\{g\}$. Then via Variation 1.20 the inequality just shown implies that $\left|f_{t} \sum_{j=1}^{\infty} t_{j} y_{j}\right|>\sum_{j=1}^{\infty}\left|t_{j}\right|$ for some $f_{t} \in A$. If we define $T(t)=\sum_{j=1}^{\infty} t_{j} y_{j}$ then we have

(i) for each $t \in l^{1}$ there is an $f_{t} \in A$ such that $\left|f_{t} T(t)\right| \geqq\|t\|_{l^{1}}$.

If $Q$ is the Minkowski functional of $A_{0}$ then $|f(x)| \leqq Q(x)$ for each $x \in E$ and all $f \in A$. Now from (i) it follows that $|Q T(t)| \geqq$ $\|t\|_{l^{1}}$. $\quad T$ is continuous by Lemma 2.2 and relatively open and oneto-one by Lemma 2.3. Thus the subspace $T\left(l^{1}\right)$ of $E$ is topologically isomorphic to $l^{1}$.

From Theorem 2.7 and Corollary 2.5 we get the following.

CoROLlaRY 2.8. Let $E$ be a $\omega$-complete barrelled space sith $_{2}$ an unconditional Schauder basis. Then if $E^{*}$ is separable, the basis is shrinking.

THEOREM 2.9. Let $E$ be either a $\omega$-complete locally convex space $_{2}$

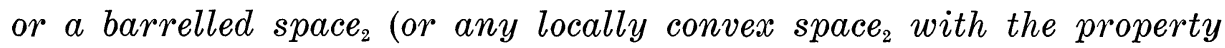
that weak* bounded subsets of $E^{*}$ are strongly bounded) and let $E$ have an unconditional Schauder basis $\left\{x_{k}, f_{k}\right\}$. If $E^{*}$ is weakly $\omega$-complete (i.e., $w\left(E^{*}, E^{* *}\right)$ - $\omega$-complete) then $\left\{x_{k}, f_{k}\right\}$ is a shrinking basis.

Proof. $\left\{f_{k}\right\}$ is a weak* unconditional basis so for each $f \in E^{*}$ the set of unordered partial sums $\left\{S_{\sigma}^{*}(f): \sigma \in \Phi\right\}$ is weak* bounded and consequently strongly bounded because of the conditions on $E$. $E^{*}$ is weakly $\omega$-complete so by Corollary $1.16, \sum_{k=1}^{\infty} f\left(x_{k}\right) f_{k}$ is strongly unconditionally convergent; and since we know the series is weak* convergent to $f$, it must be strongly unconditionally convergent to $f$.

To get a converse (Theorem 2.12 or 2.13) of Theorem 2.9 we impose on $E$ (or $E^{*}$ ) more restrictions which are provided by Theorem 2.11, a generalization of Day's Theorem 2 [2, p. 74].

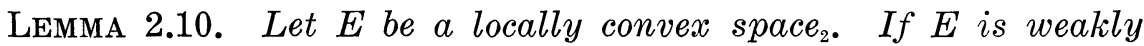
sequentially complete, then $E$ is a non-c $c_{0}$ space.

Proof. Suppose that $E$ is weakly $\omega$-complete but that there is a subspace $F$ which is topologically isomorphic to $c_{0}$. As a complete subset of the Hausdorff space $E, F$ is closed and, since it is convex, 
weakly closed. Thus $F$ is weakly $\omega$-complete which is impossible since $c_{0}$ is not weakly $\omega$-complete.

THEOREM 2.11. Let $E$ be a barrelled space . $_{2}$ with an unconditional Schauder basis $\left\{x_{k}, f_{k}\right\}$. Then the statements below are equivalent.

(1) The basis is boundedly complete.

(2) $E$ is weakly $\omega$-complete.

(3) $E$ is a $\omega$-complete non- $c_{0}$ space.

Proof. (1) $\rightarrow(2)$. Let $\left\{y_{n}\right\}$ be a weakly Cauchy sequence in $E$. Then $\left\{y_{n}\right\}$ is bounded and $\left\{f\left(y_{n}\right)\right\}_{n=1}^{\infty}$ is convergent for every $f \in E^{*}$. For each $k \in \omega$ let $a_{k}=\lim _{n \rightarrow \infty} f_{k}\left(y_{n}\right)$. Since $S_{\sigma}\left(y_{n}\right)-\sum_{k \in \sigma} a_{k} x_{k}=$ $\sum_{k \in \sigma}\left(f_{k}\left(y_{n}\right)-a_{k}\right) x_{k}, s_{\sigma}\left(y_{n}\right)$ converges with $n$ to $\sum_{k \in \sigma} a_{k} x_{k}$ (given a neighborhood of zero $V$ choose the circled neighborhood $W$ such that $W+\cdots+W \subset V$ where there are as many $W^{\prime}$ 's as the cardinal number of $\sigma$, and then pick $\varepsilon>0$ so that $\varepsilon x_{k} \in W$ for each $k \in \sigma$; for $n$ large enough, $\left|f_{k}\left(y_{n}\right)-a_{k}\right|<\varepsilon$ for all $k \in \sigma$ and so for such $n$, $\left.\sum_{k \in \sigma}\left[f_{k}\left(y_{n}\right)-a_{k}\right] \cdot x_{k} \in W+\cdots+W \subset V\right)$. Thus if $U$ is a neighborhood of zero and $V$ a barrel such that $V+V \subset U$ we have for each $\sigma \in \Phi$, an integer $N_{\sigma}$ such that $\sum_{k \in \sigma} a_{k} x_{k}-S_{\sigma}\left(y_{N_{\sigma}}\right) \in V . \quad V_{b}$ is a neighborhood of zero (see Theorem 1.18) and since $\left\{y_{n}\right\}$ is bounded there is a scalar $s>1$ such that $y_{n} \in s V_{b}$ for all $n$. Hence $S_{\sigma}\left(y_{n}\right) \in s V$ for all $\sigma \in \Phi$ and $n \in \omega$. Then for each $\sigma \in \Phi$ we have $\sum_{k \in \sigma} a_{k} x_{k} \in$ $S_{\sigma}\left(y_{N_{\sigma}}\right)+V \subset s V+V \subset s U$, proving that $\left\{\sum_{k \in \sigma} a_{k} x_{k}\right\}_{\sigma \in \mathscr{D}}$ is bounded. Since $\left\{x_{k}, f_{k}\right\}$ is boundedly complete there is a $y \in E$ such that $a_{k}=$ $f_{k}(y)$ for each $k \in \omega$, and recalling the definition of $a_{k}$ we have $\lim _{n \rightarrow \infty} f_{k}\left(y_{n}-y\right)=0$ for each $k \in \omega$.

We will prove that $y$ is the weak sequential limit of $\left\{y_{n}\right\}$. Suppose it is not. Then $u_{n}=y_{n}-y$ does not tend weakly to $\theta$ and so there is an $h \in E^{*}$, an $\varepsilon>0$, and a subsequence $\left\{z_{m}\right\}$ of $\left\{u_{n}\right\}$ such that

(i) $h\left(z_{m}\right)>\varepsilon$ for each $m \in \omega$.

In accordance with Variation 1.20 let $B$ be the equicontinuous subset of $E^{*}$ corresponding to $\{h\}$. By the result of the first paragraph of proof $\lim _{m \rightarrow \infty} S_{\sigma}\left(z_{m}\right)=\theta$. Therefore for the neighborhood of zero $U=\varepsilon / 5[B \cup\{h\}]_{0}$ there is a positive integer $m_{0}$ such that $R_{m_{0}}\left(z_{1}\right) \in U$ and then there is a $n_{1}>m_{0}$ such that $S_{m_{0}}\left(z_{n_{1}}\right) \in U$. By induction there are increasing sequences $\left\{m_{k}\right\}_{k=0}^{\infty}$ and $\left\{n_{k}\right\}_{k=1}^{\infty}$ such that

(ii) $\left|g\left(R_{m_{k}}\left(z_{n_{k}}\right)\right)\right| \leqq \varepsilon / 5$ and $\left|g\left(S_{m_{k}}\left(z_{n_{k+1}}\right)\right)\right| \leqq \varepsilon / 5$ for all $g \in B \cup\{h\}$ and $k \in \omega$.

For each positive integer $k$ define $w_{k}=\sum_{k=m_{k-1}+1}^{m_{k}} f_{k}\left(z_{n_{k}}\right) x_{k}$. Since $z_{n_{k}}-w_{k}=S_{m_{k-1}}\left(z_{n_{k}}\right)+R_{m_{k}}\left(z_{n_{k}}\right)$ we have, using (ii),

(iii) $\left|g\left(w_{k}\right)-g\left(z_{n_{k}}\right)\right| \leqq 2 \varepsilon / 5$ for all $g \in B \cup\{h\}$ and $k \in \omega$. 
Now using (i) and (iii) we have $\left|h\left(w_{k}\right)\right| \geqq\left|h\left(z_{n_{k}}\right)\right|-\mid h\left(z_{n_{k}}\right)-$ $h\left(w_{k}\right) \mid>\varepsilon-2 \varepsilon / 5$. Thus

(iv) $\left|h\left(w_{k}\right)\right|>3 \varepsilon / 5$ for each $k \in \omega$.

Let $F$ be the subspace of $l^{1}$ of finitely nonzero sequences. If $t=\left\{t_{k}\right\} \in F$ then define $T(t)=\sum_{k=1}^{\infty} t_{k} w_{k}$. For $t \neq 0$ pick the scalars $\varepsilon_{k}$ so that $\varepsilon_{k} t_{k} h\left(w_{k}\right)=\left|t_{k} h\left(w_{k}\right)\right|$; then, $\left|h\left(\sum_{k=1}^{\infty} \varepsilon_{k} t_{k} w_{k}\right)\right|=\sum_{k=1}^{\infty}\left|t_{k}\right| \cdot\left|h\left(w_{k}\right)\right|>$ $3 \varepsilon / 5 \sum_{k=1}^{\infty}\left|t_{k}\right|$. By the generalized Gurevic Theorem there must be a $g_{t} \in B$ such that $\left|g_{t}\left(\sum_{k=1}^{\infty} t_{k} w_{k}\right)\right|>3 \varepsilon / 5 \cdot \sum_{k=1}^{\infty}\left|t_{k}\right|$.

(v) For each $t \in F$ there is a $g_{t} \in B$ such that $\left|g_{t}(T(t))\right| \geqq 3 \varepsilon / 5\|t\|$.

Define $H(t)=\sum_{k=1}^{\infty} t_{k} z_{n_{k}}$ for each $t \in F$. From (iii) we get $|g(H(t)-T(t))| \leqq 2 \varepsilon / 5\|t\|$ for all $g \in B$; hence

$$
|g(H(t))| \geqq|g(T(t))|-\frac{2 \varepsilon}{5}\|t\| .
$$

Now using (v) we have

(vi) for $t \in F$ there is a $g_{t} \in B$ such that $\left|g_{t}(H(t))\right| \geqq \varepsilon / 5\|t\|$.

If $Q$ is the Minkowski functional of $B_{0}$ then $|g(x)| \leqq Q(x)$ for each $x \in E$ and all $g \in B$. Thus in view of (vi) we have $|Q(H(t))| \geqq$ $\varepsilon / 5\|t\|$ for every $t \in F$. It now follows from Lemmas 2.2 and 2.3 that $H$ is a topological isomorphism from $F$ into $E$.

Let $\overline{H(F)}$ be the closure of $H(F)$ in the completion $\hat{E}$ of $E$. Then $\overline{H(F)}$ is topologically isomorphic to $l^{1}$, and hence is weakly $\omega$-complete. Since the weakly Cauchy sequence $\left\{z_{n_{k}}\right\}$ is contained in $H(F)$, it is weakly convergent to an element $z$ of $\hat{E}$. Therefore $\left\{u_{n}\right\}$ is weakly convergent to $z$ and we have for each $k \in \omega, f_{k}(z)=$ $\lim _{n \rightarrow \infty} f_{k}\left(u_{n}\right)=0$ by the final result of the first paragraph of proof. This implies that $z=\theta$ and hence $\left\{u_{n}\right\}$ does converge weakly to $\theta$, contradicting the original assumption that it does not.

$(2) \rightarrow(3)$. Lemma 2.10.

$(3) \rightarrow(1)$. If $\left\{x_{k}\right\}$ is not boundedly complete then there is a sequence $\left\{a_{k}\right\}$ such that $D=\left\{\sum_{k=1}^{\infty} a_{k} x_{k}\right\}_{n=1}^{\infty}$ is bounded but the series does not converge and hence is not Cauchy since $E$ is $\omega$-complete. Thus there is an equicontinuous subset $A$ of $E^{*}$ and increasing sequences $\left\{m_{k}\right\}$ and $\left\{n_{k}\right\}$ with $m_{k+1}>n_{k}$ such that $y_{k} \equiv \sum_{j=m_{k}}^{n_{k}} a_{j} x_{j} \notin A_{0}$ for all $k \in \omega$. Hence, for each positive integer $k$ there is a $g_{k} \in A$ such that

(i) $\left|g_{k}\left(y_{k}\right)\right|>1$.

Let $F$ be the subspace of $c_{0}$ of finitely nonzero sequences. Define $T: F \rightarrow\left[y_{k}\right]$ as follows: $T(t)=\sum_{k \in \sigma} t_{k} y_{k}$ where $\sigma$ is the set of subscripts on which $t$ is not 0 . We shall show that $T$ is continuous. Since $\left\{x_{k}\right\}$ is unconditional and $D$ is bounded, the set of unordered partial sums $\left\{\sum_{k \in o} a_{k} x_{k}: \sigma \in \Phi\right\}$ is bounded (if $V$ is a barrel then $V_{b}$ is also a neighborhood of zero and hence there is a scalar $s$ such that $\sum_{k=1}^{n} a_{k} x_{k} \in s V_{b}$ for all $n \in \omega$; it follows that $\sum_{k \in o} a_{k} x_{k} \in s V$ for all 
$\sigma \in \Phi)$. Hence if $B_{0}$ is a neighborhood of zero ( $B$ is equicontinuous) there is a number $M$ such that $\left|f \sum_{k \in \sigma} a_{k} x_{k}\right|<M$ for all $f \in B$ and $\sigma \in \Phi$. Let $\rho=\mathbf{U}_{k \in o}\left\{j: m_{k} \leqq j \leqq n_{k}\right\}$; then $\left|\sum_{k \in o} f\left(y_{k}\right)\right|=\left|f \sum_{k \in \sigma} y_{k}\right|=$ $\left|f \sum_{j \in \sigma} a_{j} x_{j}\right|<M$. Therefore $\sum_{k \in \sigma}\left|f\left(y_{k}\right)\right| \leqq 4 M$ for all $f \in B$ and $\sigma \in \Phi$. Now suppose $t \in F$ and $\|t\|<1 / 4 M$. If $f \in B$ then $|f(T(t))|=$ $\left|\sum_{k \in \sigma} t_{k} f\left(y_{k}\right)\right| \leqq \sum_{k \in \sigma}\left|t_{k}\right| \cdot\left|f\left(y_{k}\right)\right| \leqq\|t\| \cdot \sum_{k \in \sigma}\left|f\left(y_{k}\right)\right|<1$. Therefore $T(t) \in B_{0}$, and we have proved that $T$ is continuous.

Let $t$ be a nonzero member of $F$ and pick $j$ so that $\left|t_{j}\right|=\|t\|$. Now choose $\left\{\alpha_{k}\right\}$ so that $\alpha_{k} t_{k} g_{j}\left(y_{k}\right)=\left|t_{k} g_{j}\left(y_{k}\right)\right|$. Then $\left|g_{j} \sum_{k \in o} \alpha_{k} t_{k} y_{k}\right|=$ $\left|\sum_{k \in \sigma} \alpha_{k} t_{k} g_{j}\left(y_{k}\right)\right|=\sum_{k \in \sigma}\left|t_{k}\right| \cdot\left|g_{j}\left(y_{k}\right)\right| \geqq\left|t_{j}\right| \cdot\left|g_{j}\left(y_{j}\right)\right|>\left|t_{j}\right|=\|t\|$. If $B$ is the equicontinuous set corresponding to $A$ then by the Generalized Gurevic Theorem there must be an $h_{t} \in B$ such that

(ii) $\left|h_{t} T(t)\right|>\|t\|$.

If $Q$ is the Minkowski functional of $B_{0}$ then $|h(x)| \leqq Q(x)$ for all $x \in E$ and $h \in B$. Therefore, $|Q(T(t))| \geqq\|t\|$ for each $t \in F$. By Lemma 2.3 $T$ is one-to-one and open and so $T$ is a topological isomorphism of $F$ onto $\left[y_{k}\right]$. Thus their completions, $c_{0}$ and $\overline{\left[y_{k}\right]}$, are topologically isomorphic.

A converse to 2.9 can now be proved. If $\left\{x_{k}, f_{k}\right\}$ is an unconditional shrinking basis in a barrelled space $E$, then $\left\{f_{k}\right\}$ is a strongly unconditional, boundedly complete basis for $E^{*}(2.1$ and 1.5). Therefore if $E^{*}$ is a barrelled space (in the strong topology) then by Theorem $2.11, E^{*}$ is weakly $\omega$-complete. Thus we have proved the following.

THEOREM 2.12. Let $E$ be a barrelled space ${ }_{2}$ with an adjoint $E^{*}$ which is also barrelled, and suppose that $E$ has an unconditional basis $\left\{x_{k}, f_{k}\right\}$. If $\left\{x_{k}, f_{k}\right\}$ is shrinking, then $E^{*}$ is weakly $\omega$-complete.

If the adjoint of a locally convex metrizable space is separable (in the strong topology) then $E^{*}$ is a bound space and hence barrelled [10, p. 217, 22.17]. Thus as a corollary to 2.12 we have the following.

THEOREM 2.13. Let $E$ be a barrelled metrizable space with an unconditional basis $\left\{x_{k}, f_{k}\right\}$. If $\left\{x_{k}, f_{k}\right\}$ is shrinking, then $E^{*}$ is weakly $\omega$-complete.

We include a summary of the results on shrinking bases.

CoROLlary 2.14. Let $E$ be a locally convex space ${ }_{2}$ with an unconditional Schauder basis $\left\{x_{k}, f_{k}\right\}$. Consider the following six conditions.

(1) $\left\{f_{k}, \pi\left(x_{k}\right)\right\}$ is a Schauder basis for the dual space $E^{*}$.

(2) $\left\{f_{k}, \pi\left(x_{k}\right)\right\}$ is a boundedly complete Schauder basis for $E^{*}$. 
(3) $\left\{f_{k}, \pi\left(x_{k}\right)\right\}$ is an unconditional Schauder basis for $E^{*}$.

(4) $E$ is a non-l $l^{1}$ space.

(5) $E^{*}$ is separable.

(6) $E^{*}$ is weakly $\omega$-complete.

Then (5) implies (4) (even if $E$ has no basis) and so each of (4) and (5) is a necessary condition for (1). (1), (2), and (3) are equivalent if $E$ is barrelled ((1) and (2) are equivalent even if the basis $\left\{x_{k}\right\}$ is conditional). Each of (4) and (5) is sufficient for (1) if $E$ is a $\omega$-complete barrelled space. (6) implies (1) if $E$ is a locally convex space such that weak* bounded sets are strongly bounded; and (1) implies (6) if $E$ is both barrelled and metrizable. Thus all six statements are eqvivalent if $E$ is a Fréchet space.

Recall (Proposition 1.6) that a barrelled space s $_{2}$ with a Schauder basis is reflexive if and only if the basis is both boundedly complete and shrinking. Also recall that a reflexive space is barrelled and its adjoint is reflexive and barrelled. Thus in view of 2.11 and 2.14 we have the following.

THEOREM 2.15. Let $E$ be a $\omega$-complete barrelled space s $_{2}$ with an unconditional Schauder basis. Consider the following conditions on $E$ and $E^{*}$.

(1) $E$ is weakly $\omega$-complete.

(2) $E$ is a non-c space.

(3) $E$ is a non-li space.

(4) $E^{*}$ is weakly $\omega$-complete.

(5) $E^{*}$ is a non-c space.

(6) $E^{*}$ is a non-l $l^{1}$ space.

If $E$ is reflexive then (1), (2), and (3) hold. If in addition $E^{*}$ is $\omega$-complete (as it will be if $E$ is a bound space) then all six statements hold. Then conjunction of (1) and (3) imply that $E$ is reflexive as does also the conjunction of (2) and (3).

\section{BIBLIOGRAPHY}

1. C. Bessaga and A. Pelczynski, A generalization of results of $R$. C. James concerning absolute bases in Banach spaces, Studia Math. 17 (1958), 165-174.

2. M. M. Day, Normed linear spaces, rev. ed., Academic Press, Inc., New York, 1962.

3. J. Dieudonné, On biorthogonal systems, Michigan Math. J. 2 (1954), 7-20.

4. J. Dieudonné and L. Schwartz, La dualité dans les espaces $(F)$ et $(L F)$, Ann. Inst. Fourier Grenoble 1 (1949), 61-101.

5. E. L. Dubinsky and J. R. Retherford, Schauder bases and Kothe sequence spaces, Trans. Amer. Math. Soc. 130 (1968), 265-280.

6. A. Grothendieck, Sur les applications linéaires faiblement compactes d'espace du type $C(K)$, Canad. J. Math 5 (1953), 169-173. 
7. L. A. Gurevic, On unconditional bases, Uspehi Matem. Nauk (N. S.) 8 (1953), 153-156.

8. R. C. James, Bases and reflexivity of Banach spaces, Ann. of Math. (2) 52 (1950), 518-527.

9. S. Karlin, Bases in Banach spaces, Duke Math. J. 15 (1948), 971-985.

10. J. L. Kelly, I. Namioka, et al., Linear topological spaces, D. Van Nostrand Co., Inc., Princeton, 1963.

11. C. W. McArthur, A convergence criterion with applications to series in locally convex spaces, Duke Math J. 34 (1967), 193-200.

12. - On a theorem of Orlicz and Pettis, Pacific J. Math. 22 (1967), 297-302. 13. J. R. Retherford, Bases, basic sequences and reflexivity of linear topological spaces, Math. Annalen 164 (1966) 280-285.

14. J. R. Retherford and C. W. McArthur, Some remarks on bases in linear topological spaces, Math. Annalen 164 (1966) 38-41.

15. L. J. Weill, Stability of bases in complete barrelled spaces Proc. Amer. Math. Soc. 18 (1967), 1045-1050.

Received April 3, 1967, Portions of this work were taken from the author's dissertation (directed by C. W. McArthur). Support was given by the National Science Foundation under NSF Grant GP-5952.

The Florida State University

Tallahasse; Florida

AND

UNIVERSity OF ALABAMA

University, Alabama 



\section{PACIFIC JOURNAL OF MATHEMATICS}

\section{EDITORS}

H. ROYDEN
Stanford University
Stanford, California

\section{R. R. PHELPS}

University of Washington

Seattle, Washington 98105
J. DUGUNDJI

Department of Mathematics

University of Southern California

Los Angeles, California 90007

\section{RICHARD ARENS}

University of California

Los Angeles, California 90024

\section{ASSOCIATE EDITORS}

E. F. BeCKenbaCh
B. H. NEUMANN

F. WOLF
K. YoshidA

\section{SUPPORTING INSTITUTIONS}

\author{
UNIVERSITY OF BRITISH COLUMBIA \\ CALIFORNIA INSTITUTE OF TECHNOLOGY \\ UNIVERSITY OF CALIFORNIA \\ MONTANA STATE UNIVERSITY \\ UNIVERSITY OF NEVADA \\ NEW MEXICO STATE UNIVERSITY \\ OREGON STATE UNIVERSITY \\ UNIVERSITY OF OREGON \\ OSAKA UNIVERSITY \\ UNIVERSITY OF SOUTHERN CALIFORNIA
}

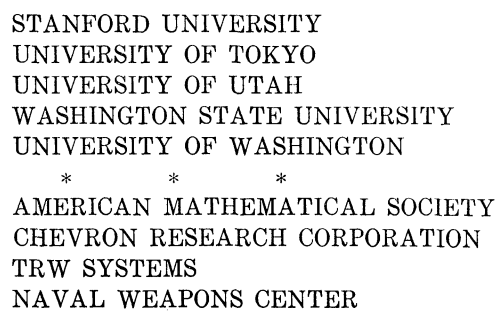

The Supporting Institutions listed above contribute to the cost of publication of this Journal, but they are not owners or publishers and have no responsibility for its content or policies.

Mathematical papers intended for publication in the Pacific Journal of Mathematics should be in typed form or offset-reproduced, double spaced with large margins. Underline Greek letters in red, German in green, and script in blue. The first paragraph or two must be capable of being used separately as a synopsis of the entire paper. It should not contain references to the bibliography. Manuscripts, in duplicate if possible, may be sent to any one of the four editors. Please classify according to the scheme of Math. Rev. 36, 1539-1546. All other communications to the editors should be addressed to the managing editor, Richard Arens, University of California, Los Angeles, California, 90024.

50 reprints are provided free for each article; additional copies may be obtained at cost in multiples of 50 .

The Pacific Journal of Mathematics is published monthly. Effective with Volume 16 the price per volume (3 numbers) is $\$ 8.00$; single issues, $\$ 3.00$. Special price for current issues to individual faculty members of supporting institutions and to individual members of the American Mathematical Society: $\$ 4.00$ per volume; single issues $\$ 1.50$. Back numbers are available.

Subscriptions, orders for back numbers, and changes of address should be sent to Pacific Journal of Mathematics, 103 Highland Boulevard, Berkeley, California, 94708.

PUBLISHED BY PACIFIC JOURNAL OF MATHEMATICS, A NON-PROFIT CORPORATION

Printed at Kokusai Bunken Insatsusha (International Academic Printing Co., Ltd.), 7-17, Fujimi 2-chome, Chiyoda-ku, Tokyo, Japan. 


\section{Pacific Journal of Mathematics \\ Vol. 29, No. $2 \quad$ June, 1969}

Bruce Langworthy Chalmers, On boundary behavior of the Bergman kernel function and related domain functionals ................... 243

William Eugene Coppage, Peirce decomposition in simple Lie-admissible power-associative rings .............................. 251

Edwin Duda, Compactness of mappings...................... 259

Earl F. Ecklund Jr., On prime divisors of the binomial coefficient......... 267

Don E. Edmondson, A modular topological lattice ............... 271

Phillip Alan Griffith, A note on a theorem of Hill ................... 279

Marcel Herzog, On finite groups with independent cyclic Sylow

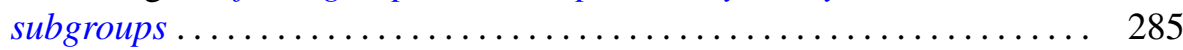

James A. Huckaba, Extensions of pseudo-valuations................. 295

S. A. Huq, Semivarieties and subfunctors of the identity functor ........ 303

I. Martin (Irving) Isaacs and Donald Steven Passman, Finite groups with small character degrees and large prime divisors. II ............ 311

Carl Kallina, A Green's function approach to perturbations of periodic

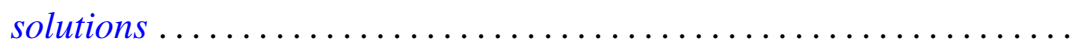

$\mathrm{Al}$ (Allen Frederick) Kelley, Jr., Analytic two-dimensional subcenter manifolds for systems with an integral ....................

Alistair H. Lachlan, Initial segments of one-one degrees ............ 351

Marion-Josephine Lim, Rank k Grassmann products ............. 367

Raymond J. McGivney and William Henry Ruckle, Multiplier algebras of

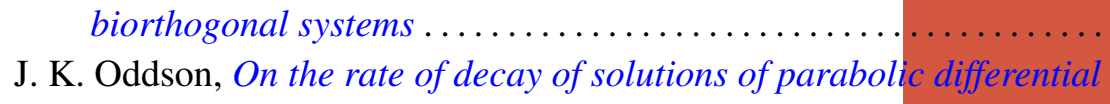

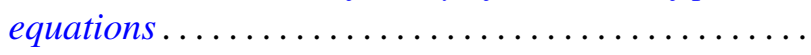

Helmut R. Salzmann, Geometries on surfaces ........... .

Annemarie Schlette, Artinian, almost abelian groups and their groups of automorphisms ............................

Edgar Lee Stout, Additional results on modules over polydisc algebras ...

Lajos Tamássy, A characteristic property of the sphere . .

Mark Lawrence Teply, Some aspects of Goldie's torsion theory. ...

Freddie Eugene Tidmore, Extremal structure of star-shaped sets ...

461

Leon Jarome Weill, Unconditional and shrinking bases in locally convex spaces... 